\title{
Anatomic Evaluation of Uterosacral and Cardinal Ligament during Robotic and Laparoscopic Surgery for Pelvic Organ Prolapse
}

\author{
Hugo H Davila1,2,3*, Renzo Di Natale, Lindsey Bruce², Lindsey Goodman², Taryn Gallo \\ ${ }^{1}$ Florida Healthcare Specialist, Urology and Minimally Invasive Surgery, Florida Cancer Specialist \& Research Institute, Sebastian \\ and Vero Beach, FL, USA \\ ${ }^{2}$ Department of Surgery, Division of Urology and Gynecology, Sebastian River Medical Center, Sebastian, FL, USA \\ ${ }^{3}$ Florida State University College of Medicine, Fort Pierce Campus, FL, USA \\ Email: *Hdavila@FLHealthcarespecialists.com
}

How to cite this paper: Davila, H.H, Di Natale, R., Bruce, L., Goodman, L. and Gallo, T. (2017) Anatomic Evaluation of Uterosacral and Cardinal Ligament during Robotic and Laparoscopic Surgery for Pelvic Organ Prolapse. Open Journal of Obstetrics and Gynecology, 7, 1216-1227. https://doi.org/10.4236/ojog.2017.712124

Received: September 5, 2017

Accepted: November 27, 2017

Published: November 30, 2017

Copyright (c) 2017 by authors and Scientific Research Publishing Inc. This work is licensed under the Creative Commons Attribution International License (CC BY 4.0).

http://creativecommons.org/licenses/by/4.0/

\begin{abstract}
Introduction: It is widely accepted that the uterosacral ligaments (UTSL), together with the cardinal ligament (CL), hold the upper vagina and cervix over the levator plate. The aim of this study is to evaluate the anatomical relationship between the right vs. left CL and UTSL during robotic and laparoscopic utero-sacral ligament suspension (UTSLS) and its implication with the surgical technique during UTSL suspension. Material and Methods: We evaluated 25 women with apical pelvic organ prolapses of stages 2 - 4 and we analyzed 100 uterosacral and cardinal ligaments. They were assigned (non-randomly) to: a) robotic-assisted laparoscopic uterosacral ligament suspension (RAL-UTSLS), b) robotic-assisted single-site utero-sacral ligament suspension (RASS-UTSLS) or laparo-endoscopic single site utero-sacral ligament suspension (LESS-UTSLS). We evaluated the length (distance between origins and insertions) of the aforementioned ligaments using the Da Vinci Si and other laparoscopic instruments like calipers. Results: The mean length of the UTSL in their caudal-cranial extent was $3.5 \pm 0.5 \mathrm{~cm}$ (right side) and $2.58 \pm 0.3 \mathrm{~cm}$ (left side). Measurements were performed on the same way for the CL, resulting in $5.1 \pm 0.3 \mathrm{~cm}$ (both side). The only significant difference was observed when comparing the right vs. left UTSL. This anatomic difference translates to $5 \pm 1$ suture stitches on the right UTSL vs. $2 \pm 1$ on the left UTSL. Conclusion: In our evaluation on cardinal and uterosacral ligament, the right UTSL was significantly longer as compared to the left and this allowed us to take 3 additional stitches on the right UTSL vs. left during RAL-UTSLS. Future studies are necessary to compare females with/without POP.
\end{abstract}




\section{Keywords}

Apical Prolapse, Vaginal Prolapse, Pelvic Anatomy, Robotic Surgery, Pelvic Floor, Pelvic Ligaments

\section{Introduction}

The prevalence of pelvic organ prolapse (POP) widely varies across studies, depending on the population studied and entry criteria. In the Women's Health Initiative study, investigators found $41 \%$ prevalence of POP at a standard physical examination in postmenopausal women older than 60 years who had not had a hysterectomy [1]. Approximately, 300,000 surgeries are annually performed to correct pelvic organ prolapse in the United States at a cost of more than $\$ 1$ billion [2] [3].

Pelvic organ prolapse (POP) is caused by structural defects in the connective tissue and the muscles that support the pelvic viscera. The uterosacral ligaments have long been regarded as a part of this support system for the pelvic organs [4]. Nichols, in his book on vaginal surgery, expressed the widely held opinion that the uterosacral ligaments, together with the cardinal ligament, hold the upper vagina and cervix over the levator plate [5].

The uterosacral ligaments (UTSL) have been studied both in cadavers and during surgery [6]. However, the borders of the ligament are difficult to establish on vaginal dissection or cadavers. In order to overcome this problem, the anatomy has been evaluated with magnetic resonance imaging (MRI) [7]. Now we are evaluating the visibility and the extent of the cardinal/uterosacral ligaments during minimally invasive surgery (laparoscopic or robotic surgery). Robotic technology has some advantages over vaginal and open abdominal surgery due to the high resolution of cameras, $3 \mathrm{D}$ vision and $10 \times$ magnification available during surgery.

More recently, there have been numerous debates on the best approach to correct POP, abdominal vs. vaginal, mesh vs. no mesh surgery. Why are we still debating the best approach to correct pelvic organ prolapses? In 2008, the large number of reported adverse events with the transvaginal placement of mesh prompted the FDA to issue a Public Health Notification outlining the potential serious consequences of such placement [8]. Due to concerns regarding the safety of vaginal meshes, there was $7 \%$ decrease in mesh use during vaginal surgery for POP in the 2 years following the 2011 FDA safety communication [9]. Laparoscopic sacrocolpopexy has been adopted by many pelvic surgeons as a way to minimize surgical morbidity and quicken patient recovery [10] [11] [12]. Abdominal sacrocolpopexy (AS) has been shown to have one of the highest long-term anatomic success rates (78\% - 100\%) among procedures for pelvic organ prolapse repair [13] with minimal complications [14].

We recently published an alternative to mesh-enhanced surgery, using UTSL 
and non-absorbable sutures, with robotic and laparoendoscopic single-site approach for suspension of apical prolapses. In a follow-up of 12 months, we observed an anatomical success rate of $92 \%$ [15]. Recently, studies have revealed the central importance of apical support in holding the pelvic organs in place. Strong correlation between apex descent and prolapse size has been established in POP-Q exam [16], dynamic MRI studies [17] [18] [19] and biomechanical modeling studies [20] [21]. Anatomically, apical support is provided by the combined action of the cardinal and uterosacral ligaments [22]. The structure of these ligaments has been described based on cadaveric dissections and cross-sectional anatomy [23] [24] [25].

The abdominal approach may offer a better understating of the important structures required for apical support. One of the main advantages of the abdominal and minimally invasive approach (laparoscopic or robotic) is a better visualization of the pelvic anatomy when compared to the vaginal approach. The aim of this study is to evaluate the surgical length between right and left cardinal and uterosacral ligaments during robotic and laparoscopic UTSL suspension and correlate it with the surgical technique.

\section{Material and Methods}

A retrospective analysis was done using the data of 2 surgeons in 2 community hospitals. Twenty five women presented with vaginal apex prolapse at pelvic organ prolapse quantitative stages 2 - 3 from January 2011 to June 2017 who were at least 21 years of age and desired minimally invasive surgery. We measured 50 UTSL and $50 \mathrm{CL}(\mathrm{n}=100)$. The procedures included in the analysis were: a) robotic-assisted Laparoscopic uterosacral ligament suspension (RAL-UTSLS), b) robotic-assisted single-site utero-sacral ligament suspension (RASS-UTSLS) or laparo-endoscopic single-site uterosacral ligament suspension (LESS-UTSLS). Patients were excluded if they were not adequate candidates for general anesthesia, had a history of prior sacrocolpopexy, suspicious adnexal masses, a history of pelvic inflammatory disease, morbid obesity (body mass index (calculated as weight $(\mathrm{kg}) /(\text { height }(\mathrm{m}))^{2}$ ) of 40 or higher), or had a history of prior or concomitant surgery for rectal prolapse. All participants signed a written informed consent and underwent a standardized evaluation, including a structured urogynecologic history and physical examination with pelvic organ prolapse quantitative staging [26].

Treatment allocation was performed by the surgeons and determined by robotic equipment availability. Two attending surgeons, Hugo H Davila (HHD) and Taryn Gallo (TG), enrolled all patients and were the primary surgeons in all procedures (assisted by other gynecologists). All attending surgeons were Board Certified and completed training in Urology (HHD) and Gynecology (TG). They were experienced in both laparoscopic and robotic procedures. The corresponding author (HHD) has experience in LESS surgery. All received formal training by Intuitive Surgical Inc in robotic single-site surgery. 
All participants received general anesthesia and underwent standard operative care. A precise description of our technique for RASS-UTSLS or RAL-UTSLS was published previously [15]. Each LESS-UTSLS and RASS UTSLS was performed using the following sutures: [1] V-Loc (Covidien), and we reinforced these absorbable sutures with [2] GORE-TEX Suture (Gore Medical) non- absorbable. When hysterectomy was done together with RASS-UTSLS or RAL -UTSLS, a supracervical hysterectomy (SCH) was performed. We used the intuitive access and Gel-POINT Mini (Applied Medical, Rancho Santa Margarita, CA) for RASS-UTSLS. A four-trocar configuration was used in both access systems: a) intuitive access: two $5 \mathrm{~mm}$, one $8 \mathrm{~mm}$ and one $10 \mathrm{~mm}$ trocars, b) GelPOINT Mini consisted of four $10 \mathrm{~mm}$ trocars. We followed our surgical technique for UTSLS and this has been described in a previous publication [15].

As previous publications have shown, the uterosacral ligaments (UTSL) are visible in MRI. They have a band-like appearance and have been identified and traced on axial MRI from its origins in the sacrum and pelvic floor to its insertion on the lateral margin of the upper vagina and cervix [7]. We evaluated this area intreaoperatively to identify UTSL (Figure 1). The UTSL was defined as the portion of the ligament under the rectovaginal peritoneum, which is thicker and denser and can be identified during surgery [27]. Other publications have shown that the CL has a web-like structure, centered on the axis of the internal iliac (anterior trunk) vessels from points of origin at the cervix [25].

The length of the CL and UTSL were measured on each side intraoperatively. To measure the length of these ligaments, 2 techniques are described during surgery to identify UTSL and CL (Figure 2). The "Origin" was defined as the point where the connective tissue condensed to a band-like structure lateral to the genital tract. This condensation of connective tissue CL/UTSL had to be visible. The "Insertion" was defined as the point at the pelvic sidewall or sacrum where the Cardinal/uterosacral ligament ended (visually). Once the CL and UTSL were identified, the measurements were done without any manipulation of the vagina, cervix or uterus. The origin of the ligament on the genital tract

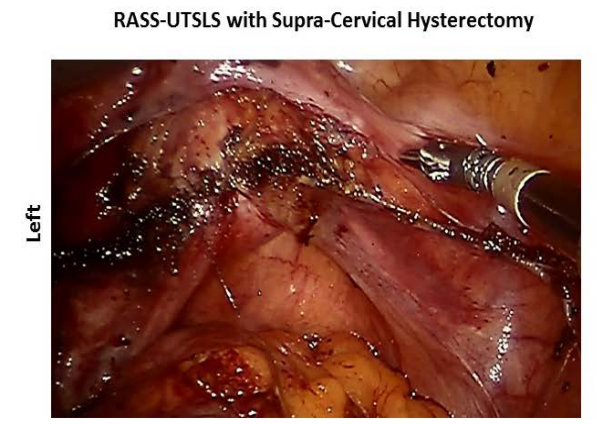
RASS-UTSLS with Supra-Cervical Hysterectomy

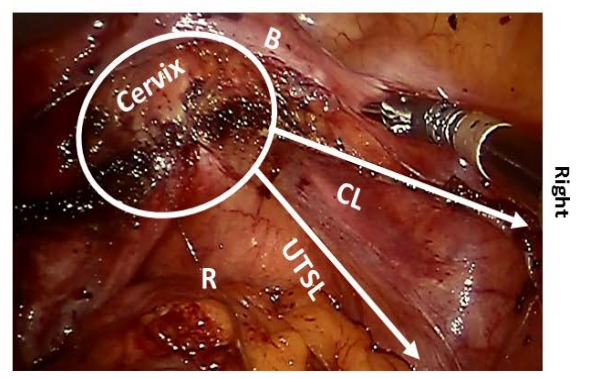

Figure 1. Images during robotic assisted single site uterosacral ligament suspension (RASS-UTSLS) after supra-cervical hysterectomy, demonstrates the uterosacral ligament (UTSL) and Cardinal Ligament (CL) origins and insertions. The origins of the UTSL and CL from the cervix (white arrows) and their direction to the sacrum or pelvic sidewall are shown. The location of the bladder (B), cervix (white circle) and rectum (R) are shown. 


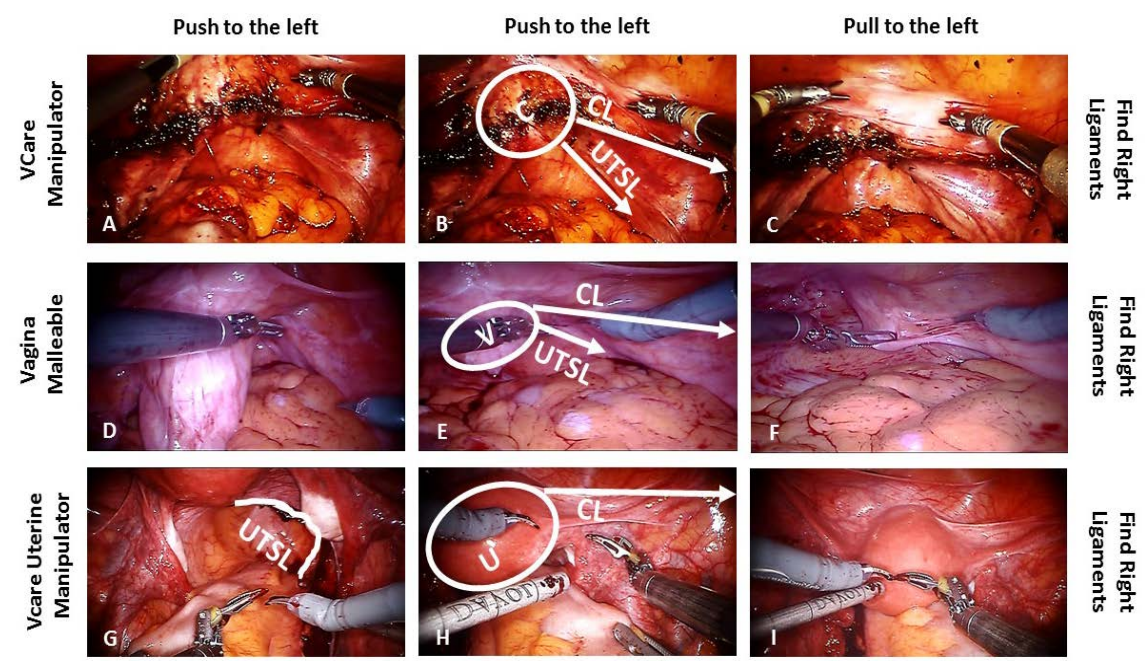

Figure 2. Identification of the uterosacral ligament (UTSL) and cardinal ligament (CL). We determined the visibility of the right ligaments by pushing to the left and pulling to the left. The location of the cervix (C); Vagina (V); Uterus (U); UTSL and CL are shown. We used a medium malleable (D) - (F) and Vcare Manipulator (ConMed, Utica NY) and Allis clamp (F); The location of the UTSL is seen on (B) (E) and (G); and CL (B) (E) and (H).

and the insertion point on the pelvic sidewall were classified as follows: from the cervix (if only cervix and no vagina was seen), from the vagina and cervix (if both were seen), or from the vagina (if only vagina and no cervix was seen).

The length (distance between origin and insertion points) measured as a tangent direction difference was assessed using the Da Vinci Si and laparoscopic instruments like calipers (Figure 3). The body axis used as reference was the caudal-cephalic axis (parallel to the rectum or vagina). All measurements were obtained and compared the left side vs. right side. The author HHD made all measurement during surgery to assess the visibility of the CL/UTSL, and their origin and insertion points. The analysis of the primary outcome (anatomic characteristics) was performed using the Student's $t$ test and included all participants who were assigned and underwent surgery. Secondary outcomes (location of the right/left ligaments, vagina, rectum and cervix) were compared using two sided Pearson's Chi-square test for dichotomous variables and two-sided Student's $t$ tests for parametric continuous variables or Wilcoxon rank-sum test for nonparametric continuous or ordinal variables. Descriptive statistics consisting of the mean, average, standard deviation, and range were calculated as appropriate.

\section{Results}

The study population had a median age ( \pm standard deviation) of $70 \pm 6$ years (range 60 - 75 years), a median parity of 2.0 (range 0 - 5), and a median body mass index of $28 \pm 5$ (range: 18 - 35); according to self-race identification, $60 \%$ 77\% were Caucasian, 23\% - 40\% African American, and 5\% other races. Current smoker status was present in $14 \%-20 \%$ and previous surgeries were present in $14 \%-20 \%$ (Table 1(a)). 

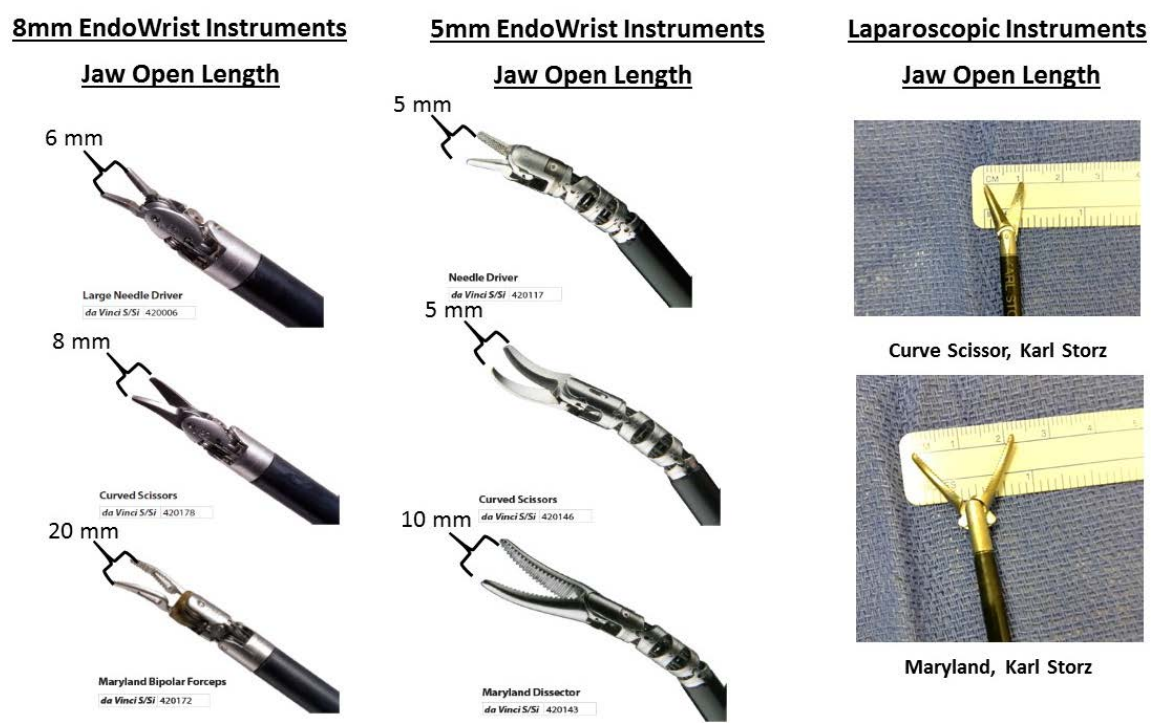

Figure 3. The length, distance between origins and insertions of the ligaments and ureters were assessed using the Da Vinci Si (5 and $8 \mathrm{~mm}$ ) and Laparoscopic (Karl Storz) instruments as calipers.

Additional procedures were done in $29 \%$ of the RASS group and 15\% LESS group (both combined with UTSL suspension), these were supracervical hysterectomy and vaginal hysterectomy, respectively. When we evaluated the surgical history in the sample, 4 (57\%) RASS-UTSLS, 9 (70\%) LESS-UTSLS and 3 (60\%) RAL-UTSLS had previous hysterectomies (Table 1(b)).

Thirty women had minimally invasive surgery, and 5 were excluded due to inability to identify and measure ligaments during the procedure. This left 25 patients for study evaluation. The cardinal $(\mathrm{n}=50)$ uterosacral ligaments were visible $(\mathrm{n}=50)$ in all patients. The following factors precluded visibility in the 5 other patients: a short distance between cervix and sacrum $(n=1)$, an enlarged uterus $(n=2)$, an overly full rectum $(n=2)$. Women in whom the cardin$\mathrm{al} /$ uterosacral ligaments were not visible did not differ from the study group regarding age or parity. In these patients we performed a robotic assisted laparoscopic sacrocolpopexy as discussed with the patients in the office and explained in the surgical consent, without ligament measuring.

The mean length ( \pm standard deviation) of the UTLS in their caudal-cephalic extent was: on the right $3.5 \pm 0.5 \mathrm{~cm}$, on the left $2.58 \pm 0.3 \mathrm{~cm}$, measured from the most caudal to the most cephalic visualization point of the UTSL (with identifiable origin and insertion points). The same measurements were done on the $\mathrm{CL}$, length was: on the right $5.1 \pm 0.3$, on the left $5.1 \pm 0.3$, calculated between the most medial to the most lateral visualization points of the CL (with identifiable origin and insertion points) Table 2(a). The only significant difference we found was when comparing UTSL lengths on right vs. left Table 2(b). Among all women, 50 origin points (UTSL) from the genital tract were found. 15 (30\%) were from the cervix, $30(60 \%)$ were from the portion of the genital tract where the cervix and vagina are together, and $5(10 \%)$ were from the vagina below the 
Table 1. (a) (b) Demographics, previous medical and surgical history. No statistically significant differences were noted between RASS UTSLs vs. LESS UTSLS vs. RAL UTSLS arms for other variables. RASS-UTSLS $(n=7)$ robotic-assisted single-site utero-sacral ligament suspension, LESS-UTSLS $(\mathrm{n}=13$ ) laparo-endoscopic single-site utero-sacral ligament suspension and RAL-UTSLS $(n=5)$ robotic-assisted laparoscopic uterosacral ligament suspension.

(a)

\begin{tabular}{ccccc}
\hline & $\begin{array}{c}\text { RASS UTSLS } \\
(\%)\end{array}$ & $\begin{array}{c}\text { LESS UTSLS } \\
(\%)\end{array}$ & $\begin{array}{c}\text { RAL UTSLS } \\
(\%)\end{array}$ & $\begin{array}{c}\text { Median } \\
(\text { SD })\end{array}$ \\
\hline N & 7 & 13 & 5 & NA \\
Age (average) & 70 & 68 & 72 & $70( \pm 6)$ \\
Parity & 2 & 2 & 2 & $2( \pm 3)$ \\
Body Mass Index (Lbs/Inches) & 28 & 28 & 29 & $28( \pm 5)$ \\
Hormone Therapy Use & 0 & $7(54)$ & $2(40)$ & 2 \\
Race & & & & \\
White & $5(71)$ & $10(77)$ & $3(60)$ & 5 \\
African American & $2(29)$ & $3(23)$ & $2(40)$ & 2 \\
Current Smoker & $1(14)$ & $4(31)$ & $1(20)$ & 1 \\
Prior Pelvic Reconstructive Surgery & $1(14)$ & $2(15)$ & $1(20)$ & 1 \\
\hline
\end{tabular}

(b)

\begin{tabular}{ccccc}
\hline & RASS UTSLS (\%) & $\begin{array}{c}\text { LESS UTSLS } \\
(\%)\end{array}$ & $\begin{array}{c}\text { RAL UTSLS } \\
(\%)\end{array}$ & Total \\
\hline N & $7(28 \%)$ & $13(52 \%)$ & $5(20 \%)$ & $100 \%$ \\
Prior Hysterectomy & $4(57 \%)$ & $9(70 \%)$ & $3(60 \%)$ & 16 \\
Supra-Cervical Hysterectomy & $2(29 \%)$ & 0 & 0 & 2 \\
Vaginal Hysterectomy & 0 & $2(15 \%)$ & 0 & 2 \\
Uterus Preservation & $1(14 \%)$ & $2(15 \%)$ & $2(40 \%)$ & $\mathbf{5}$ \\
& & & & $\mathbf{2 5}$
\end{tabular}

cervix. These three sites are the typical regions of origin of the UTSL and constitute an example of interindividual variability. Dedicated comparisons between the left and right UTSL side in 25 women showed a longer right UTSL in all of them $25(100 \%)$. This anatomical difference translates to a surgical technique finding, more suture stitches were done from the cervix or vagina to the right UTSL than the left UTSL (Table 2(c)). The pelvic organ prolapse quantitative (POP-Q) stage at baseline was similar among the groups (stages $2-3$ ). There was no evidence of recurrence at 6 months after surgery. All these findings are summarized in Table 3.

\section{Discussion}

The UTSL originates from the posterolateral aspect of the cervix at the level of the internal cervical os and from the lateral vaginal fornix [24]. Other publications described uterosacral fibers which were attached to the fascia covering the 
Table 2. Surgical length of the uterosacral ligament (UTSL, $n=50)$ and cardinal ligament $(C L, n=50)$. (a) average length of the UTSL and CL in patients with prior hysterectomy, during supra-cervical hysterectomy, vaginal hysterectomy and uterus preservation; (b) Multivariate analysis of surgical length of the UTSL and CL Right vs. Left. The right UTSL was significantly longer than the left $(P$ value $<0.05)$; (c) Multivariate analysis of number of suture stitches of the UTSL Right vs. Left. We did more stitches in the right UTSL and this difference was significant as compared to the left $(P$ value $<0.05)$.

(a)

\begin{tabular}{ccccccc}
\hline UTSL and CL Length & $\begin{array}{c}\text { Prior } \\
\text { Hysterectomy }\end{array}$ & $\begin{array}{c}\text { Supra-Cervical } \\
\text { Hysterectomy }\end{array}$ & $\begin{array}{c}\text { Vaginal } \\
\text { Hysterectomy }\end{array}$ & $\begin{array}{c}\text { Uterus } \\
\text { Preservation }\end{array}$ & $\begin{array}{c}\text { Average UTSL } \\
\text { Length }\end{array}$ & $\begin{array}{c}\text { Average CL } \\
\text { Length }\end{array}$ \\
\hline N & 16 & 2 & 2 & 5 & NA & NA \\
$\begin{array}{c}\text { Surgical Length Right } \\
\text { UTSL (cm) }\end{array}$ & 3.00 & 3.50 & 4.00 & 3.50 & $3.50( \pm 0.5)$ & NA \\
$\begin{array}{c}\text { Surgical Length Left UTSL } \\
\quad(\mathrm{cm})\end{array}$ & 2.30 & 3.00 & 2.50 & 2.50 & $2.58( \pm 0.3)$ & NA \\
$\begin{array}{c}\text { Surgical Length Right CL } \\
\text { (cm) }\end{array}$ & 4.8 & 5.4 & 4.8 & 5.4 & NA & $5.1( \pm 0.3)$ \\
$\begin{array}{c}\text { Surgical Length Left CL } \\
\text { (cm) }\end{array}$ & 4.8 & 5.4 & 4.8 & 5.4 & NA & $5.1( \pm 0.3)$ \\
\hline
\end{tabular}

(b)

\begin{tabular}{cccc}
\hline UTSL and CL Length & Average Right & Average Left & $P$ value \\
\hline $\begin{array}{c}\text { Surgical Length UTSL } \\
(\mathrm{cm})\end{array}$ & $3.50( \pm 0.5)$ & $2.58( \pm 0.3)$ & $<0.05$ \\
Surgical Length CL $(\mathrm{cm})$ & $5.1( \pm 0.3)$ & $5.1( \pm 0.3)$ & $>0.05$ \\
\hline
\end{tabular}

(c)

\begin{tabular}{cccc}
\hline Number of Suture Stitches & Average Right & Average Left & $\boldsymbol{P}$ value \\
\hline UTSL & $5( \pm 1)$ & $2( \pm 1)$ & $<0.05$ \\
CL & NA & NA & NA \\
\hline
\end{tabular}

levator ani, coccygeus, and obturator muscles, as well as the presacral fascia [23]. Our findings corroborate these macroscopic findings. Previous publications observed that the sigmoid's mesentery caused the left UTSL to appear less prominent. We found that in most women the left UTSL was shorter in its caudal-cephalic extent when compared to the right side. This may be attributed to the embryologic development of this region, which includes rotation and attachment of the sigmoid mesentery to the left pelvic side wall. This allows us to places more stitches on the right UTSL.

Other studies have used MRI to evaluate the UTSL and have shown that the origin of the UTSL from the genital tract extends from the cervix to the upper vagina. The insertion on the pelvic sidewall occurs to the sacrospinous ligament and the coccygeus muscle in $82 \%$ of all cases, but only in $7 \%$ of cases the UTSL does insert on the sacrum [7]. This suggests that the UTSL exhibits greater anatomic interindividual variability than the name implies, and this might be an important insight for the understanding of the pelvic organ support mechanism. Another concern is that UTSL may not be a true ligament.

Other publications have noted that in virtually all subjects, the CL attachment to the lateral pelvic sidewall is a triangular area located at the first branching of 
Table 3. Pre-operative anatomic data and short-term operative outcomes. There are no statistically significant differences between RASS-UTSLS vs. LESS-UTSLS vs. RAL-UTSLS at a 6-month follow-up. RASS-UTSLS robotic assisted single site uterosacral ligament suspension, LESS-UTSLS laparo-endoscopic single-site utero-sacral ligament suspension, RAL-UTSLS robotic-assisted laparoscopic utero-sacral ligament suspension.

\begin{tabular}{ccccccc}
\hline & LESS & RASS & RAL & LESS & RASS & RAL \\
& UTSLS & UTSLS & UTSLS & UTSLS & UTSLS & UTSLS \\
\hline & Baseline & Baseline & Baseline & 6 Months 6 & Months 6 Months \\
\hline $\begin{array}{c}\text { Pelvic Organ Prolapse } \\
\text { Quantitative Stage }\end{array}$ & $\mathrm{n}=13$ & $\mathrm{n}=7$ & $\mathrm{n}=5$ & $\mathrm{n}=13$ & $\mathrm{n}=7$ & $\mathrm{n}=5$ \\
0 & 0 & 0 & 0 & $7(54)$ & $3(43)$ & $3(60)$ \\
1 & 0 & 0 & 0 & $5(38)$ & $4(57)$ & $2(40)$ \\
2 & $7(54)$ & $3(42.85)$ & $2(40)$ & 0 & 0 & 0 \\
3 & $6(46)$ & $4(57.14)$ & $2(40)$ & $1(8)$ & 0 & 0 \\
4 & 0 & 0 & $1(20)$ & 0 & 0 & 0 \\
\hline
\end{tabular}

the internal iliac artery [28]. MRI studies had suggested its origin to be near the origin of the anterior trunk of the internal iliac artery [29], though this vessel was not always well visualized. In our study the overall length of the CL was 5 $\mathrm{cm}$. This measurement differs to those in other publications due to the fact that in this particular case we only measure the visible points of origin/insertion during surgery. Other publications used MRI or cadaveric dissection of the CL.

There are several limitations to consider in this study. Firstly, surgery was performed on the dorsal lithotomy position; this may not accurately reflect the pelvic floor anatomy in the upright position. Secondly, filling of the rectum might influence the appearance of the UTSL during surgery and result in length differences. A standardized bowel preparation was used in all patients to try overcoming potential interindividual differences; rectal filling did not appear to be a significant issue during ligament length measuring (rectal contents prohibited evaluation of the UTSL only in 2 patients $-0.6 \%$ of initial sample). Third, the identification of the structures was done based on direct visualization techniques alone. We cannot determine the direction of the fibers within the connective tissue and thus, cannot be certain that the ligaments directly connect to the pelvic sidewall structures. Finally, using robotic/laparoscopic instruments (like calipers) as references to perform the measurements may result in significant length variability and therefore affect the estimated total length. More accurate intraoperative techniques/instruments for length measurement should be considered in future studies.

\section{Conclusion}

The contribution of different disease mechanisms to pelvic organ prolapse needs to be better understood in order to improve functional outcomes. Abdominal approaches during minimally invasive surgery (either laparoscopic or robotic) may open a new frontier in our understanding of the pelvic floor anatomy. 
High-quality image modalities available in robotic and laparoscopic surgery can provide a better localization of these ligaments. This is a descriptive study on cardinal and uterosacral ligament. The right UTSL was significantly longer as compared to the left and this allows us to take 3 additional stitches on the right UTSL vs. left during RAL-UTSLS. Futures studies are necessary to compare females with/without POP.

\section{Funding}

None.

\section{Conflicts of Interest}

Authors have nothing to disclose.

\section{Compliance with Ethical Standards and Informed Consent}

Informed consent was obtained from all individuals included in the study.

\section{References}

[1] Hendrix, S.L., Clark, A., Nygaard, I., Aragaki, A., Barnabei, V. and McTiernan, A. (2002) Pelvic Organ Prolapse in the Women's Health Initiative: Gravity and Gravidity. American Journal of Obstetrics \& Gynecology, 186, 1160-1166. https://doi.org/10.1067/mob.2002.123819

[2] Olsen, A.L., Smith, V.J., Bergstrom, J.O., Colling, J.C. and Clark, A.L. (1997) Epidemiology of Surgically Managed Pelvic Organ Prolapse and Urinary Incontinence. Obstetrics \& Gynecology, 89, 501-506. https://doi.org/10.1016/S0029-7844(97)00058-6

[3] Subak, L.L., Waetjen, L.E., van den Eeden, S., Thom, D.H., Vittinghoff, E. and Brown, J.S. (2001) Cost of Pelvic Organ Prolapse Surgery in the United States. $O b$ stetrics \& Gynecology, 98, 646-651.

[4] Singh, K., Jakab, M., Reid, W.M., Berger, L.A. and Hoyte, L. (2003) Three-Dimensional Magnetic Resonance Imaging Assessment of Levator Ani Morphologic Features in Different Grades of Prolapse. Obstetrics \& Gynecology, 188, 910-915. https://doi.org/10.1067/mob.2003.254

[5] Nichols, D.H. and Randall, C.L. (1996) Vaginal Surgery. 4th Edition, Chapter 5, Williams \& Wilkins, Baltimore, USA, 101-118.

[6] Buller, J.L., Thompson, J.R., Cundiff, G.W., Krueger Sullivan, L., Schon Ybarra, M.A., et al. (2001) Uterosacral Ligament: Description of Anatomic Relationships to Optimize Surgical Safety. Obstetrics \& Gynecology, 97, 873-879. https://doi.org/10.1097/00006250-200106000-00002

[7] Umek, W.H., Morgan, D.M., Ashton-Miller, J.A. and DeLancey, J.O. (2004) Quantitative Analysis of Uterosacral Ligament Origin and Insertion Points by Magnetic Resonance Imaging. Obstetrics \& Gynecology, 103, 447-451. https://doi.org/10.1097/01.AOG.0000113104.22887.cd

[8] Division of Small Manufacturers, International and Consumer Assistance (DSMICA). (2011) Update on Serious Complications Associated with Transvaginal Placement of Surgical Mesh for Pelvic Organ Prolapse: FDA Safety Communication. Silver Spring (MD, USA). US Food and Drug Administration. 
https://wayback.archive-it.org/7993/20161022170630/http://www.fda.gov/NewsEve nts/Newsroom/PressAnnouncements/ucm262752.htm

[9] Sedrakyan, A., Chughtai, B. and Mao, J. (2016) Regulatory Warnings and Use of Surgical Mesh in Pelvic Organ Prolapse. JAMA Internal Medicine, 176, 275-277. https://doi.org/10.1001/jamainternmed.2015.6595

[10] Cosson, M., Rajabally, R., Bogaert, E., Querleu, D. and Crepin, G. (2002) Laparoscopic Sacrocolpopexy, Hysterectomy, and Burch Colposuspension: Feasibility and Short-Term Complications of 77 Procedures. JSLS, 6, 115-119.

[11] Nezhat, C.H., Nezhat, F. and Nezhat, C. (1994) Laparoscopic Sacral Colpopexy for Vaginal Vault Prolapse. Obstetrics \& Gynecology, 84, 885-888.

[12] Ostrzenski, A. (1996) Laparoscopic Colposuspension for Total Vaginal Prolapse. International Journal of Gynecology \& Obstetrics, 55, 147-152. https://doi.org/10.1016/S0020-7292(96)02731-2

[13] Nygaard, I.E., McCreery, R., Brubaker, L., Connolly, A.M., Cundiff, G., Weber, A.M., et al. (2004) Abdominal Sacrocolpopexy: A Comprehensive Review. Obstetrics \& Gynecology, 104, 805-823. https://doi.org/10.1097/01.AOG.0000139514.90897.07

[14] Serati, M., Bogani, G., Sorice, P., Braga, A., Torella, M., Salvatore, S., Uccella, S., Cromi, A. and Ghezzi, F. (2014) Robot-Assisted Sacrocolpopexy for Pelvic Organ Prolapse: A Systematic Review and Meta-Analysis of Comparative Studies. European Urology, 66, 303-318. https://doi.org/10.1016/j.eururo.2014.02.053

[15] Davila, H.H., Gallo, T., Bruce, L. and Landrey, C. (2016) Robotic and Laparoendoscopic Single-Site Utero-Sacral Ligament Suspension for Apical Vaginal Prolapse: Evaluation of Our Technique and Perioperative Outcomes. Journal of Robotic Surgery.

[16] Rooney, K., Kenton, K., Mueller, E.R., FitzGerald, M.P. and Brubaker, L. (2006) Advanced Anterior Vaginal Wall Prolapse Is Highly Correlated with Apical Prolapse. American Journal of Obstetrics \& Gynecology, 195, 1837-1840. https://doi.org/10.1016/j.ajog.2006.06.065

[17] Summers, A., Winkel, L.A., Hussain, H.K. and DeLancey, J.O. (2006) The Relationship between Anterior and Apical Compartment Support. American Journal of $O b$ stetrics \& Gynecology, 194, 1438-1443. https://doi.org/10.1016/j.ajog.2006.01.057

[18] Chen, L., Ramanah, R., Hsu, Y., Ashton-Miller, J.A. and DeLancey, J.O. (2013) Cardinal and Deep Uterosacral Ligament Lines of Action: MRI Based 3D Technique Development and Preliminary Findings in Normal Women. International Urogynecology Journal, 24, 37-45. https://doi.org/10.1007/s00192-012-1801-4

[19] Tunn, R., DeLancey, J.O. and Quint, E.E. (2001) Visibility of Pelvic Organ Support System Structures in Magnetic Resonance Images without an Endovaginal Coil. American Journal of Obstetrics \& Gynecology, 184, 1156-1163. https://doi.org/10.1067/mob.2001.112972

[20] Chen, L., Ashton-Miller, J.A., Hsu, Y. and DeLancey, J.O. (2006) Interaction among Apical Support, Levator ani Impairment and Anterior Vaginal Wall Prolapse. $\mathrm{Ob}$ stetrics \& Gynecology, 108, 324-332. https://doi.org/10.1097/01.AOG.0000227786.69257.a8

[21] Chen, L., Ashton-Miller, J.A. and DeLancey, J.O. (2009) A 3D Finite Element Model of Anterior Vaginal Wall Support to Evaluate Mechanisms Underlying Cystocele Formation. Journal of Biomechanics, 42, 1371-1377. https://doi.org/10.1016/j.jbiomech.2009.04.043 
[22] DeLancey, J.O. (1992) Anatomic Aspects of Vaginal Eversion after Hysterectomy. American Journal of Obstetrics \& Gynecology, 166, 1717-1784. https://doi.org/10.1016/0002-9378(92)91562-O

[23] Blaisdell, F.E. (1917) The Anatomy of the Sacro-Uterine Ligaments. The Anatomical Record, 12, 1-42. https://doi.org/10.1002/ar.1090120102

[24] Campbell, R.M. (1950) The Anatomy and Histology of the Sacrouterine Ligaments. American Journal of Obstetrics \& Gynecology, 59, 1. https://doi.org/10.1016/0002-9378(50)90334-6

[25] Range, R.L. and Woodburne, R.T. (1964) The Gross and Microscopic Anatomy of the Transverse Cervical Ligaments. American Journal of Obstetrics \& Gynecology, 90, 460. https://doi.org/10.1016/0002-9378(64)90802-6

[26] Klauschie, J.L., Suozzi, B.A., O’Brien, M.M. and McBride, A.W. (2009) A Comparison of Laparoscopic and Abdominal Sacral Colpopexy: Objective Outcome and Perioperative Differences. International Urogynecology Journal and Pelvic Floor Dysfunction, 20, 273-279. https://doi.org/10.1007/s00192-008-0768-7

[27] Butler-Manuel, S.A., Buttery, L.D., A’Hern, R.P., Polak, J.M. and Barton, D.P. (2000) Pelvic Nerve Plexus Trauma at Radical Hysterectomy and Simple Hysterectomy: The Nerve Content of the Uterine Supporting Ligaments. Cancer, 89, 834-841.

https://doi.org/10.1002/1097-0142(20000815)89:4<834::AID-CNCR16>3.0.CO;2-7

[28] Samaan, A., Vu, D., Haylen, B.T. and Tse, K. (2014) Cardinal Ligament Surgical Anatomy: Cardinal Points at Hysterectomy. International Urogynecology Journal, 25, 189-195. https://doi.org/10.1007/s00192-013-2248-y

[29] Ramanah, R., Berger, M.B., Chen, L., Riethmuller, D. and DeLancey, J.O. (2012) See It in 3D: Researchers Examined Structural Links between the Cardinal and Uterosacral Ligaments. American Journal of Obstetrics \& Gynecology, 207, 437e1-7. 